\title{
Defining favourable reference values for bird populations in Italy: setting long-term conservation targets for priority species
}

\author{
MATTIA BRAMBILLA, MARCO GUSTIN and CLAUDIO CELADA
}

\begin{abstract}
Summary
Setting favourable reference values (FRV) for conservation targets may help in assessing the conservation status of species and habitats. FRVs should be expressed through population size or demographic parameters that are likely to ensure the long-term persistence of a species across its range. We developed a conceptual framework for defining FRVs for Italian birds included in Annex I of the European Union's Wild Birds Directive 79/409/CEE. The approach was based on demographic trends, current population size and the minimum viable population concept. We subdivided bird species according to abundance at the national scale, spatial distribution in discrete isolated populations and/or biogeographic categories. FRVs based on population viability analysis (PVA) were provided for populations of less than 2,500 pairs. For species with more than 2,500 pairs and a wide, more or less continuous range, the FRV was expressed in terms of breeding density at different spatial scales for non-colonial species. Out of the 88 species considered, we were able to formulate FRVs based on PVA for 47 populations belonging to 21 species, and breeding density for 15 further species; lack of adequate data prevented us from defining FRVs for the remaining species. Further work should focus on the translation of FRV density values into population size and on the definition of reference values for range and habitat.
\end{abstract}

\section{Introduction}

The conservation status of a species can be measured through a favourable reference value (FRV) that indicates range, habitat, population size and/or other parameters likely to ensure its longterm persistence in all or part of its range. These values may be used to evaluate the conservation status of populations and they can aid effective conservation planning because species or populations whose parameters strongly deviate from FRVs may be considered more 'at risk'. Therefore, it is at least conceivable that defining reference values for a population, or a part of the range of a target species, could help the assessment and monitoring of its conservation status.

A critical issue for species conservation is population size. Below a certain threshold, the survival of an organism is likely to be compromised by several factors affecting survival and reproduction (Soule 1987). Identifying the minimum number of individuals needed to ensure the long-term persistence of a species or population, taking into account the impact of deterministic and stochastic factors, has become a common feature in conservation planning. Such an approach is known as population viability analysis (PVA), where demographic models are built to improve understanding of animal populations and/or to complement field studies in the development of conservation strategies (Katzner et al. 2006). PVAs show the impact of demographic, environmental, genetic and other factors on the dynamics of wildlife populations. Very often, PVA models are used to identify a minimum viable population (MVP; Lacy 200oa; Reed et al. 2003). PVA models often face problems when dealing with large numbers of individuals and for 
very abundant species they may result in analyses that are excessively time-consuming, less reliable, and have highly variable outputs (Lacy et al. 2005). PVA models can also help to identify 'optimal' population sizes in different situations; for example, they can identify MVPs for species facing extinction risk, or for populations large enough to ensure long-term persistence even in less favourable conditions, or populations subject to harvesting where the species is not currently threatened with extinction. PVA models can show which traits are more important for conservation, e.g. reproductive or survival rates, through elasticity and sensitivity analyses (Naujokaitis-Lewis et al. 2009).

When PVA models are unlikely to be useful or are impossible to develop due to a lack of data, other population parameters may help to identify conservation targets and assess conservation status. In particular, breeding density (the number of individuals, pairs, territories or nests per unit area) can give a first indication of the conservation status of a species, indirectly representing abundance and/or habitat suitability for a given species over a given area.

\section{Conservation targets under EU directives}

The European Union's Habitats Directive (92/43/CEE) and Wild Birds Directive (79/409/CEE) represent the main tools for EU member states to use in stemming the decline in biodiversity. The Habitats Committee (in the Directorate-General Environment within the European Commission) has defined guidelines for monitoring and reporting the conservation status of species and habitats of EU interest within the framework of Article 17 of the Habitats Directive (European Commission 2005). These guidelines require member states to evaluate the conservation status of species, to assess their demographic trends and to provide a population figure defined as a 'Favourable Reference Value' for each species. The guidelines state that an FRV should be defined for each species and habitat by each member state. According to the general principles and indications of the Habitats Directive and to our own interpretation, the FRV should indicate a population figure (or another demographic reference value, if population size is unfeasible) which is likely to ensure long-term persistence of the species in its range or a part thereof.

An FRV-based assessment of conservation status has to be completed for each species. FRVs (e.g. for range, area covered, population size) should be established on a scientific basis, based on the best available conservation knowledge and in a transparent way. 'Best expert judgement' may be used to define it in absence of other data (European Commission 2005).

Establishing FRVs must be distinguished from establishing concrete targets: setting targets is the translation of reference values into operational, practical and feasible short-, medium- and long-term targets/milestones. Member states are therefore encouraged to include FRVs in their monitoring reports, as the establishment of such values will strongly support discussions on status assessment and priority setting at the biogeographical or national level. Until now, except for a concise report on species conservation status in Denmark (Pihl et al. 2006), none of the member states of the EU has provided a comprehensive evaluation of FRVs for its breeding bird species included in Annex I of the Wild Birds Directive.

In this paper, we report a conservation planning exercise carried out at a national scale (Italy) for the definition of FRVs for bird species conservation at a population scale within the framework set by the EU Habitats Directive and Wild Birds Directive. We develop a conceptual framework for the definition of population FRVs for birds and for the establishment of FRVs as a long-term reference for conservation objectives for the Italian populations of species included in Annex I of the Wild Birds Directive.

\section{Methods}

FRVs were defined only for species regularly breeding in Italy, except for 12 species undergoing current demographic expansion after recent colonisation (30 years). We decided not to formulate 
an FRV for these species because population expansion following colonisation may lead to unpredictable outcomes in most cases.

We developed a protocol for defining FRVs based on demographic trends, current populations, and the minimum viable population concept. The entire process required one year of work and involved analysis of more than 1,90o bibliographic references.

Italian breeding birds were assigned to categories according to their distribution (species that were widespread or those that occurred in discrete populations) and abundance $(\leq 2,500$ breeding pairs or $>2,500$ breeding pairs). The cut-off value was set at 2,500 pairs as it seemed reasonable to use PVAs only for small populations, for which population modelling gives the most reliable and accurate results (Lacy et al. 2005). Species with fewer than 2,500 pairs were further divided into two groups: i) species with a single population; a single FRV for the Italian population was provided and ii) species with several non-contiguous populations, i.e. range distribution structured into discrete units (populations, colonies, or group of colonies) with virtually no gene flow between them; in this case an FRV was provided for each main unit.

Species with more than 2,500 pairs were also divided into two groups according to their distribution. The first group contained species whose range is subdivided into discrete units (populations, colonies, or group of colonies) with populations $<2,500$ breeding pairs; a specific FRV was provided for each unit. The second group included species with a large and more or less continuous range (all possible discrete units relevant from a conservation point of view, i.e. excluding units of minimal size, $>2,500$ breeding pairs); for this group, the FRV was expressed in terms of breeding density at different spatial scales for non-colonial species. An FRV is not provided for colonial species with population units $>2,500$ breeding pairs as it does not appear feasible to define a density-based reference value that may not reflect (or be correlated to) population size.

The program Vortex (version 9.72) was used to evaluate the extinction risk for species and populations with fewer than 2,500 breeding pairs (Lacy 1993, 200ob; Lacy et al. 2005). The analyses included demographic parameters, (age structure, mortality at different ages), reproductive parameters (type of reproductive system, first and maximum age of reproduction, sex ratio at birth, percentage of males and females taking part in reproduction, percentage of successful nests and/or number of young fledged) and environmental parameters (occurrence of catastrophes, carrying capacity of the habitat), as requested by modelling procedures in Vortex. Species and populations with fewer than 2,500 breeding pairs were divided into three groups.

The first group included species or populations that showed a constant positive trend (increase, or increase alternating with/followed by stability) during the last 20-30 years. For this group, using known parameters for each species, we calculated the probability of extinction $(P)$ using the highest estimate of the current population (e.g. taking 200 when the estimated population is 100-200 pairs). If $P \leq 0.01$ (no more than $1 \%$ chance of extinction) in the next 100 years, the estimated population is taken as the FRV for the given species or population; if $P>0.01$ (probability of extinction greater than $1 \%$ ) we calculated the minimum viable population (MVP) as the population showing a $99 \%$ probability of persistence over 100 years $(P=0.01$ in the next 100 years) and took this as the FRV for that species or population. Whenever possible, different simulations were carried out with varying parameters within the range of known values for a given species.

The second group included species or populations with unfavourable or unknown trends. In this case, we calculated the population with $1 \%$ probability of extinction over the next 100 years using the less favourable values for breeding and reproductive parameters found in the literature (but avoiding extreme values linked to exceptionally unfavourable years or conditions) and took that population figure as the FRV. Such a population figure should ensure species persistence even under less favourable scenarios.

The third group included species or populations with a high probability of extinction in the next 100 years; we calculated the MVP (population with $1 \%$ probability of extinction in the next Ioo years) using the average demographic and breeding parameters known for the species and 
took this as the FRV. The unfavourable conditions shown by these species/populations made it unrealistic to calculate an MVP using the less favourable values.

Calculating FRVs requires a large set of demographic and reproductive parameters that are crucial for population modelling analyses. Therefore, an FRV was calculated only when the main parameters needed for population analysis were known. In some cases, data for closely related species belonging to the same genus and with comparable ecology and breeding density in suitable habitats were used instead (e.g. Katzner et al. 2006). Otherwise, an FRV was not calculated. When only one or a few of the parameters required by population modelling were lacking, we carried out simulations varying the above parameters within the range observed for closely related species and analysing variation in the results of the simulation procedure.

All simulations were conducted using the environmental variation coefficient (EV) or the standard deviation reported for a given species. When a specific EV or standard deviation was not available, we adopted an EV equal to $20 \%$ of the value of the parameter considered. When simulations were run on the less favourable scenarios, variation was set at zero. When reproductive parameters were entered in the form of productivity (number of fledglings per pair, irrespective of whether pairs were successful or unsuccessful), we considered $100 \%$ of adults as taking part in reproduction, with the only exception being species in which a proportion of adults do not participate in reproduction (i.e. do not try to breed, as is the case with several large raptor species). One type of catastrophe, with a frequency of $1 \%$ and associated with a $50 \%$ reduction in both reproductive outputs and survival, was entered into all simulations, except where stated. All parameter values have been specified in the species factsheets.

For abundant, non-colonial species with fewer than 2,500 breeding pairs, with no evidence for subdivision of populations into discrete units, we considered available breeding density data. When optimal habitat patches and related breeding density were known, we reported them as 'optimal' density, thus empirically obtaining or approaching a maximum value. At the largest scale, habitat quality is likely to vary spatially, and we aimed to obtain a 'favourable' density. Reference values for breeding density were always expressed in the unit area of the original data (e.g. if densities for a given species were available as the number of pairs $\mathrm{km}^{-2}$, the FRV was similarly expressed as pairs $\mathrm{km}^{-2}$ ). We mostly focused on breeding densities reported for Italy, but also considered data from other European countries.

Methods and case studies were then shown to experienced conservation biologists and ornithologists to get an expert-based revision and validation of both general procedures (methodological issues) and specific results (individual species outputs).

Here, we report the outcome of the process and present three different examples to show how FRVs were calculated in practical terms by using population modelling techniques and known estimates of breeding density.

\section{Results}

\section{Outcomes of the process}

Of the 88 Italian breeding species included in the Wild Birds Directive, one (European Dotterel Charadrius morinellus) was excluded because it is not a regular breeder (Brichetti and Fracasso 2004). We calculated an FRV based on population modelling analyses for 47 populations (with fewer than 2,500 pairs) belonging to 21 species. The FRV was higher than current population estimates for all these species and populations, except (i) populations of Golden Eagle Aquila chrysaetos in areas where the species is believed to have reached carrying capacity and (ii) all main populations of Peregrine Falco peregrinus, which currently appear to be levelling out around the likely carrying capacity, and the species is colonising urban habitats (Allavena and Brunelli 2003, Brunelli 2007).

For a further 15 species we provided FRVs in terms of breeding density at one or more scales, mainly depending on habitat patchiness and territory size. All FRVs formulated are reported in Appendix $\mathrm{S}_{I}$ in the online Supplementary Materials. 
For two species occurring in Italy with $<2,500$ pairs (Honey Buzzard Pernis apivorus and Short-toed Eagle Circaetus gallicus), data on reproductive and/or demographic parameters were scarce or lacking, while data on breeding density were fairly abundant. Therefore, we suggested favourable breeding densities, but were not able to formulate an FRV.

For 12 species that recently colonised or recolonised Italy, some after reintroduction (first nesting attempt no more than 30 years ago) we did not provide an FRV. The same applied to recently established populations of other species (e.g. reintroduced populations of Griffon Vulture Gyps fulvus in mainland Italy and Sicily). However, in four cases (three populations of Griffon Vulture and the Alpine population of Bearded Vulture Gypaetus barbatus) we provided conservation targets for reintroduced populations, defined as MVPs based on currently measured values of main demographic and reproductive parameters. We also did not provide an FRV for three colonial breeders with all populations or colonies $>2,500$ breeding pairs: Cory's Shearwater Calonectris diomedea, Black-crowned Night Heron Nycticorax nycticorax and Little Egret Egretta garzetta.

The remaining 35 species all have at least one population for which an FRV could be calculated (through population modelling or breeding density), but calculation was prevented by: a significant and largely unquantified exchange of individuals with extra-national populations (Audouin's Gull Larus audouinii); unsustainable current mortality rates (Griffon Vulture in Sardinia), or a lack of adequate data (all others). Lack of data particularly affected certain families, especially the Rallidae (crakes: four species belonging to the genera Porzana, Crex and Porphyrio) and Picidae (woodpeckers: five species belonging to the genera Picus, Dendrocopos, Dryocopus, Picoides). The number of species falling into each category is summarised in Figure 1 .

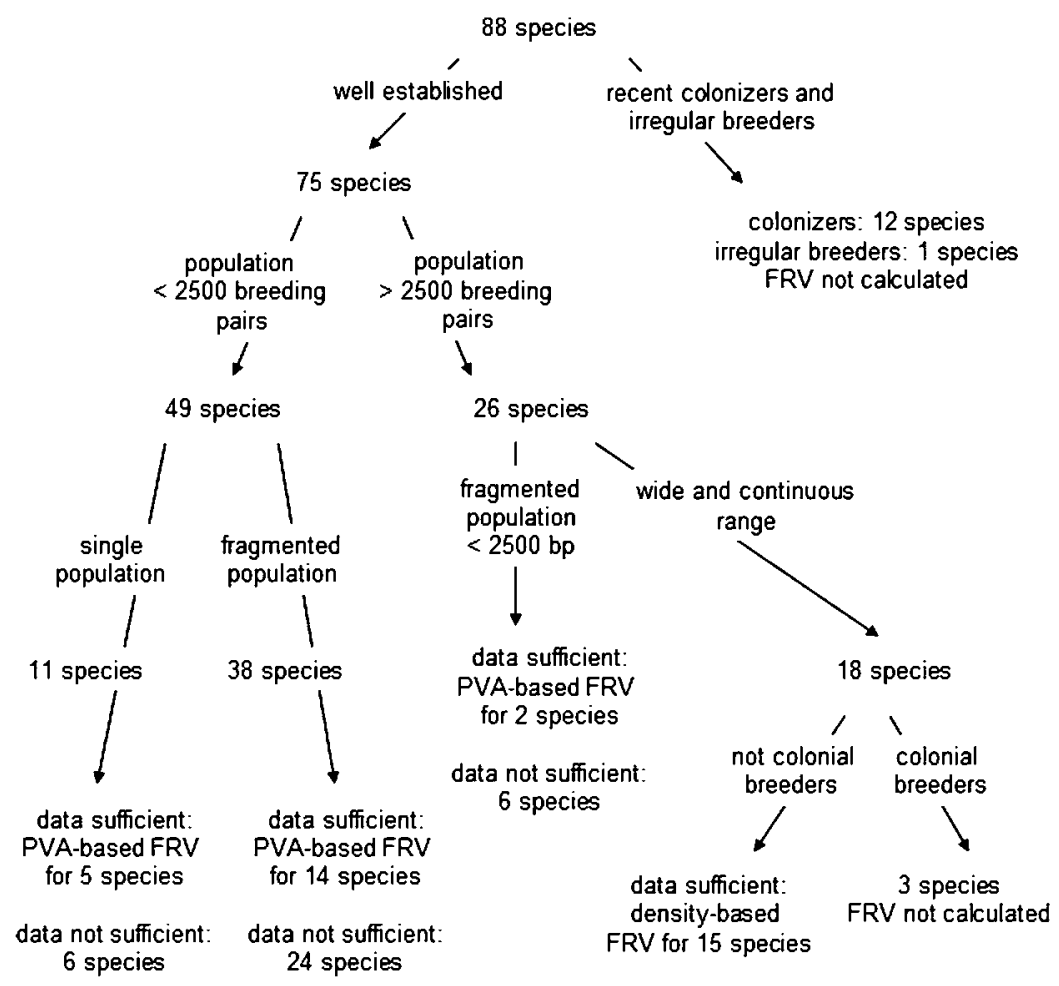

Figure 1 . Procedure followed to define FRVs according to species characteristics and outcomes of the process. 


\section{Worked examples}

Population modelling techniques: Egyptian Vulture Neophron percnopterus and Eleonora's Falcon Falco eleonorae

\section{Here we provide two examples of an FRV calculated through PVA:}

One species with a single population for which a single FRV is given (Egyptian Vulture) and one species with different FRVs for each discrete population (Eleonora's Falcon).

The Egyptian Vulture is a rare raptor, which in Europe mainly inhabits Mediterranean countries, especially Spain. Its current distribution in Italy essentially consists of only one population in Sicily (Brichetti and Fracasso 2003), estimated at only seven pairs (Ceccolini and Cenerini 2008). Egyptian Vulture is clearly one of the most endangered breeding species in Italy, being close to extinction, and thus belongs to the third group of species or populations treated under a PVA approach, i.e. those with a high probability of extinction in the next 1oo years. Therefore, we calculated the MVP (extinction probability $1 \%$ in the next 100 years) using the average demographic and breeding parameters known for the species and took that as the FRV. We carried out different simulations, incorporating mortality and breeding parameters from different studies, in order (i) to account for the contrasting data reported for that species, and (ii) to show how the long-term viability of the target population is strongly dependent on both adult survival and breeding success. We developed two different scenarios: the first considered mortality values taken from a stable population (Donázar et al. 2002) and the second took values calculated for a steeply declining population (Inigo et al. 2008). In the first scenario, we used the following parameter values: age of first reproduction five years; maximum life expectancy 20 years; productivity $0.97 \pm 0.25$ (Sarà and Di Vittorio 2003); proportion of breeding females equal to 0.9 (we assumed that not all females mate each year because of the very limited number of individuals); mortality equal to $50 \%$ in the first year of life, $40 \%$ in the second, $30 \%$ in the third, $10 \%$ in the fourth, $5 \%$ in the fifth, and $3 \%$ for adults, deriving values from Donázar et al. (2002) but using adult mortality of $3 \%$ instead of $<2 \%$ (too low for the declining Italian population). Carrying capacity was set to mirror the Sicilian breeding population of the 1970 (40 pairs, 132 individuals; Ceccolini et al. 2006). In the second scenario, we modified parameter values in accordance with recent Spanish data (Inigo et al. 2008): age of first breeding of six years, mortality equal to $27 \pm 2 \%$ in the first and second years of life, $22 \pm 3 \%$ in the third, fourth and fifth years, $40 \pm 5 \%$ in the sixth year, and $17 \pm 2 \%$ in adults. In this scenario, we did not include catastrophes.

First scenario. The current population has a probability of extinction around $90 \%$ in the next Ioo years. A population equal to carrying capacity showed a quite high extinction risk (extinction probability $P \sim 17 \%$ in 100 years) and a tendency to decrease. Keeping constant mortality values, a population equal to the carrying capacity ( 132 individuals) has an extinction probability $P \sim 1 \%$ with an increase in breeding success, which should be equal to $78 \%$ of adults breeding successfully, with a fledging rate of 1.4 young per successful pair. Similar breeding parameter values fall well within the range of values reported from other European countries (breeding success between 74.1\% and 96.5\% and productivity between 0.8 and 1.5; Braillon 1979, 1987, Bergier and Cheylan 1980, Marco and Garcia 1981, Bergier 1985, Gallardo et al. 1987, Vasconcelos 1987, Donázar and Ceballos 1988, Abuladze and Shergalin 1998). We assumed that similar values could also be reached in Italy through protection of breeding sites and food supply for pairs experiencing low food availability. Therefore, we proposed an FRV (subject to adult mortality of $3 \%$ ) of 4 o pairs, with breeding success of $78 \%$ and fledging rate of 1.4 .

Second scenario. Because of the extremely high adult mortality ( $17 \%$ instead of $3 \%$ ), the risk of extinction further increased, and the current population would become extinct within the next 40 years, even without catastrophes or inbreeding effects and with an initial population of individuals all between five and nine years of age (a particularly favourable situation). However, with such low adult survival it is virtually impossible to maintain a viable population, even with 
higher breeding success. With adult mortality equal to $6 \pm 2 \%$ (intermediate between the values reported by Donázar et al. 2002, and Inigo et al. 2008, but closer to the former) and keeping other parameters constant, the long term survival probability becomes higher and population trend becomes stable. In short, barring catastrophes and inbreeding depression, a population of 132 individuals may be viable in the long term (extinction probability $=1 \%$ in 100 years) with a breeding success of $80 \%$, fledging rate equal to 1.4, adult mortality of $6 \%$ (and mortality for other age classes as in the second scenario). With catastrophes as default in the other simulation, the probability of extinction of this hypothetical population would increase to $15 \%$. The simulations carried out under the second scenario clearly show the need to keep adult mortality as low as possible in Egyptian Vultures, which have low annual productivity but a potentially long reproductive phase (Inigo et al. 2008).

The Egyptian Vulture example shows how the calculation of an FRV for a single population could also be based on different scenarios in order to consider the importance of improving different parameters, such as breeding success instead of mortality, to make it possible for a population currently threatened with extinction to reach carrying capacity and to survive in the long term.

Eleonora's Falcon is an endemic Mediterranean bird of prey that inhabits rocky islands and islets (Cramp and Simmons 1977). The current distribution of the species in Italy essentially includes two populations, the larger one inhabiting Sardinia and adjacent smaller islands, and the other occupying Sicily and especially its satellite islands, the Eolian and Pelagie islands (Brichetti and Fracasso 2003). The Sardinian population is currently estimated at about 500 pairs, the Sicilian population at 138-204 pairs (Gustin et al. 2005). The Sicilian population appears to have declined in recent years (Corso 2008), while the apparently positive population trend shown by the species in Sardinia is arguably due to improved knowledge of the distribution and size of the colonies (Gustin et al. 2005). Therefore, Eleonora's Falcon populations are considered to have an unfavourable (Sicilian) or unknown (Sardinian) trend and the main parameter values selected were among the less favourable. Since there is no data on carrying capacity, this is prudently assumed to be equal to the initial tested population in both cases. The only data on demographic parameters were reported by Ristow et al. (1989) and are as follows: mortality $78 \%$ before adulthood, $13 \%$ in adulthood (two years); maximum longevity at least 16 years; first breeding generally takes place at two years of age for females and three years for males, but exceptions are numerous.

Cramp and Simmons (1977) reported first breeding at two years of age, without differentiating between the sexes. Exceptionally, one-year-old individuals can breed (Ristow et al. 1989). We thus decided to treat individuals of two years of age or older as breeders, following the analysis by Ristow et al. (1989) of mortality and demographic structure in this species. We thus estimate the following mortality rates per year: $65 \%$ during the first year of life, $37 \%$ during the second (amounting to an overall mortality of $78 \%$ before breeding) and $13 \%$ from the third year on. Considering all known productivity values in Italy, the mean productivity is equal to 1.26 ( \pm 0.39 SD) fledglings per pair (Badami 1995a,b, Brichetti and Fracasso 2003, Gustin et al. 2005, Medda 2006, Spina and Leonardi 2007). The Sardinian population (500 pairs, equivalent to 1,250 individuals under a stable-age distribution), which has good chances for long-term survival given current average productivity, would face a high risk of extinction should its productivity fall to 1.1 or less, something which has happened more than once in the past (Gustin et al. 2005, Medda 2006, Corso 2008). In this case, the MVP $(P=1 \%)$ would be 2,200 individuals, or 900 pairs. This figure, which would guarantee survival even under scenarios slightly less favourable than the current one, is therefore proposed as the FRV for the Sardinian population.

The Sicilian population (average estimate 176 pairs, or about 425 individuals) shows a relatively high $(>10 \%)$ probability of extinction over the next 100 years; the MVP $(P=1 \%)$ calculated using the figure for average productivity recorded in Italy would be 800 individuals or about 320 pairs; we thus propose 320 pairs as the FRV for the Sicilian population, on condition that average adult productivity is not below 1.26. Ristow and Wink (1985) reported that a productivity of 1.2 fledged young for each breeding attempt was necessary to maintain a colony, a figure that accords with the results of this analysis. 
The example of Eleonora's Falcon shows how calculation of the FRV for different populations is based on different scenarios in order to reflect different conservation objectives: reaching a population size able to survive even in worsened conditions (Sardinia), or a population size with reasonably good chances of long-term persistence, in the case of smaller, and currently threatened, populations (Sicily).

\section{Known estimates of breeding density:}

Woodlark Lullula arborea and Red-backed Shrike Lanius collurio. Woodlark is a quite widespread but rarely abundant passerine species endemic to Europe. It has a rather large population in Italy (estimated at 20,000-40,000 pairs; Brichetti and Fracasso 2007) which lacks appreciable subdivision into discrete units or populations, although the decline of the Alpine population is now increasing the gap between this and the other parts of the Italian range. However, the ecology of this species is quite similar throughout its range and densities appear similar in different areas. Therefore, the FRV is formulated in terms of breeding density at two spatial scales. Considering the highest known densities, optimal Woodlark density at large scales can be identified as to pairs $\mathrm{km}^{-2}$ and at a local scale equal to three pairs to ha ${ }^{-1}$ (Brambilla and Rubolini 2009). Ten pairs $\mathrm{km}^{-2}$ and three pairs per 10 ha are thus proposed as FRVs at 'large' and 'local' scales, respectively.

Red-backed Shrike is a typical passerine species of Eurasian low-intensity farmland landscapes. It underwent large population declines over most of Europe during the last century (Heath 1994). The Italian population is estimated at 50,000-120,000 pairs (BirdLife International 2004). There have been many studies of its breeding ecology (see Brambilla et al. 2009 and references therein) and thus estimates of breeding densities for Italy and elsewhere are numerous and relate to different habitats, from highly suitable landscapes to patchy habitats with lower average suitability (Casale and Brambilla 2009). Reviewing the data, we found that in areas with mosaics of open and semiopen habitats, breeding densities could be around one pair $\mathrm{km}^{-2}$ at large scales, but in mostly open habitats (large pastures, low-intensity farming systems, etc.) densities up to five pairs $\mathrm{km}^{-2}$ are frequently reached (Brambilla et al. 2007, 2009). At the local scale (< 100 ha), in mosaic areas (with scattered patches of suitable open or semi-open habitats), density should be no lower than 0.5 pairs 1o ha ${ }^{-1}$ (Brambilla and Casale 2008). Densities in suitable habitats are 3-5 pairs $10 \mathrm{ha}^{-1}$ and reach 8-10 pairs 1o ha ${ }^{-1}$ in exceptionally suitable areas (Brambilla et al. 2009). Therefore, we proposed an FRV value of one pair $\mathrm{km}^{-2}$ at 'large' scales (for mosaic areas with scattered suitable habitats; five pairs $\mathrm{km}^{-2}$ for open or semi-open areas) and 0.5 pairs $10 \mathrm{ha}^{-1}$ at 'local' scales (for mosaic areas; five pairs 1o $\mathrm{ha}^{-1}$ for suitable habitats; 10 pairs $10 \mathrm{ha}^{-1}$ for exceptionally suitable areas).

\section{Discussion}

When defining FRVs for breeding bird species in Italy, we assigned species to multiple categories, reflecting differences in abundance, distribution and demographic trends.

For species that colonised Italy in the last 30 years (or recolonised spontaneously or were reintroduced), we did not provide an FRV. We believe that the dynamics of population expansion following colonisation may lead to unpredictable outcomes for most of those species, especially in the absence of reliable estimates of the carrying capacity of the habitats. However, it was possible to calculate an MVP for some species using current estimates of demographic and reproductive parameters and such a value was proposed as the mid-term conservation target; this procedure was applied to Bearded Vulture and to three reintroduced populations of Griffon Vulture. Setting concrete targets means translating reference values into operational, practical and feasible short-, medium- and long-term targets. For all other species, an FRV could be obtained with adequate knowledge of the parameters. Filling the gap in knowledge that affects quite a large number of families (especially some poorly studied ones, such as the Rallidae and Picidae) truly represents a conservation priority, as it is impossible to set reference values for such poorly known species. In 
fact, demographic parameters were the most frequently lacking data, but reproductive data were also unknown for a large number of species.

The use of density-based FRVs allowed us to by-pass the impossibility of obtaining reliable estimates of population size for most of the abundant species and the weakness of PVAs with a large number of individuals. A simple comparison between ground-measured density values at a site or group of sites and the reference value proposed for target species should allow site managers to make an immediate rough assessment of the conservation status of target bird species. The use of density values (especially for passerines) may hopefully become a practical tool for evaluating population status and for deriving consequent implications under management plans of Natura 2000 sites and other protected areas.

\section{Conclusions}

Our work aimed to establish long-term conservation targets in the form of 'Favourable Reference Values', i.e. demographic values which should guarantee species survival in the long term, for bird species of particular conservation interest at national and EU levels. Obviously, maintaining populations of adequate size is not independent of habitat and general environmental conditions, which should be maintained to the necessary extent and quality needed by target species. Ideally, formulation of FRVs for range and habitat should accompany the population reference value, but at present, relevant data are lacking for most bird species.

Unfortunately, lack of adequate knowledge prevented the definition of FRVs for some of the target species. On the other hand, our work indicated the principal areas where further research is necessary.

Additional work should focus on translating density values into population estimates on the basis of the current, historic, and/or potential range of those species; in order to do so, however, potentially suitable habitat should be quantified, but these data are currently lacking. A further potential problem related to the use of breeding density as a reference value is that sites functioning as ecological traps may host a high density of a species even though they act as population sinks (Battin 2004). Data on reproductive success may demonstrate a real correlation between breeding density and local status/dynamics. However, breeding density is widely adopted as an indirect measure of habitat suitability and it could be taken as a first step in measuring conservation status for our general purposes, aimed at monitoring species at a large scale.

Despite these limitations, our approach provides a useful framework for defining a population reference value for species of varying conservation concern, accounting for their demographic history, life-history characteristics and distributional features.

\section{Acknowledgements}

We are particularly grateful to all the people who contributed to this work, and especially to scientists who revised our general approach to FRV, both in Italy (N. Baccetti, G. Boano, G. Bogliani, P. Brichetti, P. Pedrini, D. Rubolini, F. Spina) and at BirdLife International (B. Barov, I. Burfield). An anonymous reviewer provided very useful suggestions on a first draft. We are very grateful also to V. Bergero, E. Bianchi, G. Bottari, F. Casale, P. Colombani, E. Duprè, R. Falco, F. Piccarolo, M. Ravasini for kind cooperation. LIPU received funding for this project from MATTM (Ministry of the Environment, Territorial Protection, and the Sea); ISPRA and FLA participated as project partners.

\section{References}

Abuladze, A. and Shergalin, J. (1998) The Egyptian Vulture (Neophron percnopterus) in the former USSR. Pp. 183-195 in R. D. Chancellor, B. Meyburg and J. J. Ferrer, eds. Holarctic birds of prey. Merida and Berlin: Adenex and World 
Working Group on Birds of Prey and Owls.

Allavena, S. and Brunelli, M. (2003) Revisione delle conoscenze sulla distribuzione e consistenza del Pellegrino Falco peregrinus in Italia. Avocetta 27: 20-23.

Badami, A. (1995a) Note sulla biologia riproduttiva del falco della regina. Avocetta 19: 106.

Badami, A. (1995b) Relazioni tra fattori ecologici e riproduzione nel falco della regina (Falco eleonorae). Suppl. Ric. Biol. Selvaggina 22: 115-119.

Battin, J. (2004) When good animals love bad habitats: ecological traps and the conservation of animal populations. Conserv. Biol. 18: 1482-1491.

Bergier, P. (1985) La reproduction du Vautour Percnoptère Neophron percnopterus en Provence (SE France) de 1979 à 1983. Bull. World Working Group on Birds of Prey and Owls 2: $77-78$.

Bergier, P. and Cheylan, G. (1980) Status, succès de reproduction et alimentation $\mathrm{du}$ vautour percnoptère (Neophron percnopterus) en France méditerranéenne. Alauda 48: 75-97.

BirdLife International (2004) Birds in the European Union: a status assessment. Wageningen, The Netherlands: BirdLife International.

Braillon, B. (1979) Le Percnoptere dans les Pyrénées francaises. Cahiers Université Pau Pays de l'Adour 1: 319-329.

Braillon, B. (1987) La nidification du Vautour Percnoptère Neophron percnopterus sur le versant Nord des Pyrénées, un suivì d'ensemble commencé il y a 27 ans. Acta Biol. Montana 7: 101-113.

Brambilla, M. and Casale, F. (2008) L'Averla piccola Lanius collurio come specie guida per la gestione naturalistica degli ambienti agricoli. Relazione tecnica finale. Technical report. Milano, Italy: Fondazione Lombardia per l'Ambiente.

Brambilla, M. and Rubolini, D. (2009) Intraseasonal changes in distribution and habitat associations of a multi-brooded bird species: implications for conservation planning. Anim. Conserv. 12: 71-77.

Brambilla, M., Casale, F., Bergero, V., Crovetto, G. M., Falco, R., Negri, I.,
Siccardi, P. and Bogliani, G. (2009) GISmodels work well, but are not enough: Habitat preferences of Lanius collurio at multiple levels and conservation implications. Biol. Conserv. 142: 2033-2042.

Brambilla, M., Rubolini, D. and Guidali, F. (2007) Between land abandonment and agricultural intensification: habitat preferences of Red-backed Shrikes Lanius collurio in low-intensity farming conditions. Bird Study 54: 160-167.

Brichetti, P. and Fracasso, G. (2003) Ornitologia Italiana. Vol. 1-Gaviidae-Falconidae. Bologna, Italy: Oasi Alberto Perdisa Editore.

Brichetti, P. and Fracasso, G. (2004) Ornitologia Italiana. Vol. 2-Tetraonidae-Scolopacidae. Bologna, Italy: Oasi Alberto Perdisa Editore.

Brichetti, P. and Fracasso, G. (2007) Ornitologia Italiana. Vol. 4-Apodidae-Prunellidae. Bologna, Italy: Alberto Perdisa Editore.

Brunelli, M. (2007) Lo stato delle ricerche sul Pellegrino Falco peregrinus in Italia. Pp. 50-57 in M. Magrini, P. Perna and M. Scotti, eds. Aquila reale, lanario e pellegrino nell'Italia peninsulare - Stato delle conoscenze e problemi di conservazione. Atti del Convegno, Serra San Quirico (Ancona), 26-28 marzo 2004. Parco Regionale Gola della Rossa e di Frasassi.

Casale, F. and Brambilla, M. (2009) Averla piccola. Ecologia e conservazione. Milano, Italy: Fondazione Lombardia per l'Ambiente e Regione Lombardia.

Ceccolini, G. and Cenerini, A. (2008) La tecnica dell'hacking sulla specie Capovaccaio, il bilancio di quattro anni di attività. Pp. 30-37 in F. Bellini, N. Cillo, V. Giacoia. and M. Gustin, eds. L'avifauna di interesse comunitario delle gravine ioniche. Laterza: Oasi LIPU Gravina di Laterza.

Ceccolini, G., Cenerini, A. and Sarà, M. (2006) Il Capovaccaio, specie prossima all'estinzione? Pp. 351-365 in M. Fraissinet and F. Petretti, eds. Salvati dall'Arca. Bologna: Alberto Perdisa Editore.

Corso, A. (2008) Progetto Falco della regina Falco eleonorae: Anno 2008. Eolie e Lampedusa. Parma, Italy: LIPU.

Cramp, S. and Simmons, K. E. L. (1977) The birds of the western Palearctic. Volume I. Oxford, UK: Oxford University Press. 
Donázar, J. A. and Ceballos, O. (1988) Alimentacion y tasas reproductoras del Alimoche (Neophron percnopterus) en Navarra. Ardeola 35: 3-14.

Donázar, J. A., Palacios, C. J., Gangoso, L., Ceballos, O., González, M. J. and Hiraldo, F. (2002) Conservation status and limiting factors in the endangered population of Egyptian vulture (Neophron percnopterus) in the Canary Islands. Biol. Conserv. 107: 89-97.

European Commission (2005) Assessment, monitoring and reporting of conservation status - Preparing the 2001-2006 report under Article 17 of the Habitats Directive. Note to the Habitats Committee, 15 March 2005. Brussels: European Commission, Directorate-General Environment.

Gallardo, M., Astruy, J. C., Cochet, G., Seriot, J., Neri, F. Torre, J. and Thibault, J. C. (1987) Gestion des populations de grands rapaces. Rev. Ecol. 4: 241-252.

Gustin, M., Corso, A. and Medda, M. (2005) Monitoring on breeding population of Eleonora's Falcon Falco eleonorae in Italy during 2005. Pp. $1-51$ in Conservation measures for Falco eleonorae in Greece. LIFE Nature Project LIFEo3 NAT/GR/oooog1.

Heath, M. F. (1994) Red-backed Shrike Lanius collurio. Pp: 410-411 in G. M. Tucker and M. F. Heath. Birds in Europe: their conservation status. Cambridge, UK: BirdLife International (BirdLife International Conservation Series no. 3).

Inigo, A., Barov, B., Orhun, C. and Gallo-Orsi, U. (2008) Species action plan for the Egyptian Vulture Neophron percnopterus percnopterus in the European Union. Prepared by SEO and BirdLife International on behalf of the European Commission.

Katzner, T. E., Bragin, E. A. and MilnerGulland, E. J. (2006) Modelling populations of long-lived birds of prey for conservation: a study of imperial eagles (Aquila heliaca) in Kazakhstan. Biol. Conserv. 132: 322-335.

Lacy, R. C. (1993) VORTEX: a computer simulation model for population viability analysis. Wildl. Res. 20: 45-65.

Lacy, R. C. (200oa) Considering threats to the viability of small populations using individual-based models. Ecol. Bull. 48: 39-51.
Lacy, R. C. (200ob) Structure of the VORTEX simulation model for population viability analysis. Ecol. Bull. 48: 191-203.

Lacy, R. C., Borbat, M. and Pollak, J. P. (2005) VORTEX: A stochastic simulation of the extinction process. Brookfield, IL: Chicago Zoological Society.

Marco, J. and Garcia, D. (1981) Situation actuelle des populations de nècrophages (Gyps fulvus, Gypaetus barbatus, et Neophron percnopterus) en Catalogne. Rapaces Méditerranéens: 1: 119-129.

Medda, M. (2006) Stagione riproduttiva del Falco della regina (Falco eleonorae) nell'isola di San Pietro (Sardegna sud-occidentale). Annual report 2006. Unpublished Report to LIPU (Lega Italiana Protezione Uccelli) Pp: $1-15$.

Naujokaitis-Lewis, I. R., Curtis, J. M. R., Arcese, P. and Rosenfeld, J. (2009) Sensitivity analyses of spatial population viability analysis models for species at risk and habitat conservation planning. Conserv. Biol. 23: 225-229.

Pihl, S., Clausen, P., Laursen, K., Madsen, J. and Bregnballe, T. (2006) Conservation status of bird species in Denmark covered by the EU Wild Birds Directive. Copenhagen: Ministry of Environment, National Environmental Research Institute. (NERI Technical Report No. 570).

Reed, D. H., O'Grady, J. J., Brook, B. W., Ballou, J. D. and Frankham, R. (2003) Estimates of minimum viable population sizes for vertebrates and factors influencing those estimates. Biol. Conserv. 113: 23-34.

Ristow, D. and Wink, M. (1985) Breeding success and conservation management of Eleonora's Falcon. Pp. $147-152$ in I. Newton and R. D. Chancellor, eds. Conservation studies on raptors. Proceedings of the II World Conference on Birds of Prey. (ICBP Technical Publication No. 5).

Ristow, D., Scharlau, W. and Wink, M. (1989) Population structure and mortality of Eleonora's Falcon Falco eleonorae. Pp. 321-326 in B.-U. Meyburg and R. D. Chancellor, eds. Raptors in the modern world. Berlin, London and Paris: WWGBP.

Sarà, M. and Di Vittorio, M. (2003) Factors influencing the distribution, abundance and nest-site selection of an endangered Egyptian 
vulture (Neophron percnopterus) population in Sicily. Anim. Conserv. 6: 317-328.

Soulé, M. E., ed. (1987) Viable populations for conservation. Cambridge, UK: Cambridge University Press.

Spina, F. and Leonardi, G., eds. (2007) Piano $\mathrm{d}$ 'azione nazionale per il falco della regina Falco eleonorae. Rome: Ministerio del-
l'Ambiente, Instututo Nazionale per la Fauna Selvatica. (Quaderni di Conservazione della Natura 26).

Vasconcelos, M. (1987) Le dynamique des populations de necrophages (Gyps fulvus et Neophron percnopterus) au fleuve tejo international. Suppl. Ric. Biol. Selvaggina 12: $287-294$.

\section{MATTIA BRAMBILLA*}

Fondazione Lombardia per l'Ambiente, Settore Biodiversità e Aree Protette, Piazza Diaz 7, I-20123 Milano, Italy.

Lega Italiana Protezione Uccelli (LIPU), Department of Nature Conservation, via Trento 49, I-43100, Parma, Italy.

MARCO GUSTIN, CLAUDIO CELADA

Lega Italiana Protezione Uccelli (LIPU), Department of Nature Conservation, via Trento 49, I-4310o, Parma, Italy.

*Author for correspondence; email: brambilla.mattia@gmail.com

Received 31 August 2009; revision accepted 12 March 2010;

Published online 28 July 2010 